\title{
Desarrollo pondoestatural y maduración esquelética en niños con disfunción cerebral mínima. (Comunicación preliminar.)
}

Es un hecho conocido que niños con retardo mental, parálisis cerebral y otras alteraciones severas de la función del Sistema Nervioso Central, muestran con frecuencia retardo del desarrollo pondoestatural. ${ }^{(1,2)}$ Sin embargo, los retardados mentales constituyen un grupo heterogéneo y las alteraciones de su desarrollo físico varían según las diferentes categorías etiológicas, siendo más marcadas en aquellos con malformaciones cerebrales, encefalopatías congénitas y daño cerebral perinatal..$^{(3)}$

Utilizando las tablas de maduración esquelética de Schmid, ${ }^{(4)}$ se describieron anormalidades en el desarrollo esquelético de la mano y muñeca en el $71 \%$ de un grupo heterogéneo de 233 niños con daño cerebral, especialmente retraso de la maduración ósea. ${ }^{(5)}$ Posteriormente, Lyttkens y Persson, ${ }^{(6)}$ en Suecia, examinaron las radiografías de carpo en $\mathbf{4 8}$ niños con daño cerebral y en 48 controles sanos, encontrando que había retardo de la maduración ósea en una proporción significativa de niños con daño cerebral (23\%), comparados con los controles $(4 \%)$. La frecuencia de talla baja fue también. mucho mayor en el grupo con daño cerebral (44\%) que en el grupo control (10\%). El grupo índice de estos autores incluía casos leves y severos, con un rango que abarcaba desde niños con alteraciones conductuales de origen presumiblemente orgánico hasta otros con parálisis cerebral abierta.

Recientemente, Oettinger y colaboradores analizaron $^{(7)}$ las radiografías de carpo de un

\footnotetext{
* Unidad de Neurología, Depto. de Pediatría, H. Roberto del Rio. Becado en Neurología Pediátrica, Universidad de British Columbia, Canadá.
}

grupo de 53 niños de inteligencia normal, diagnosticados como portadores de disfunción cerebral mínima, según el criterio de Clements. ${ }^{(8)} \mathrm{La}$ edad ósea de este grupo estaba significativamente retardada al ser comparada con las normas norteamericanas de Greulich y Pyle. ${ }^{(9)}$ Estos autores concluyen que la evolución de la edad ósea podría añadir otro parámetro al estudio de los niños que presentasen síntomas y signos atribuibles a disfunción cerebral mínima. Al investigar la maduración esquelética de estos niños, debe tenerse presente que se ha demostra$\left.\mathrm{do}^{(10}, 11\right)$ disminución del crecimiento en pacientes hipercinéticos tratados con dosis altas de drogas estimulantes, particularmente dextroanfetamina. El crecimiento de niños hiperactivos sin tratamiento con estimulantes es aparentemente normal.(11)

\section{MATERIAL Y METODO}

En la Clínica de Desarrollo Neurológico del Hospital de Niños de Vancouver, Canadá, hemos seleccionado 60 niños con diagnóstico de disfunción cerebral mínima que tenían un estudio completo tanto clínico como de laboratorio, incluyendo radiografías de carpo y mano para examen de su edad ósea. Nos planteamos como objetivo del análisis de este grupo de niños, el de averiguar si presentaban diferencias significativas de maduración esquelética, como también de peso, talla y perímetro craneano, al ser comparados con normales. Secundariamente, hemos tratado de investigar la existencia de subgrupos dentro del amplio síndrome de "disfunción cerebral minima", que pudieran presentar retar- 
do de la maduración ósea como característica diferencial.

El diagnóstico de disfunción cerebral mínima fue hecho por un equipo que incluía neurólogo-pediatra, psiquiatra, psicólogo clínico y psicopedagogo, además de fisioterapeuta y terapeuta ocupacional. El criterio diagnóstico fue esencialmente el de Clements, ${ }^{(8)}$ modificado por Crichton:(12) "niños de inteligencia normal, que presentan problemas conductuales, de aprendizaje, de lenguaje y de integración sensoriomotriz, asociados a desviaciones leves del examen neurológico y de la maduración motora".

Nuestro grupo estaba constituido por 45 niños y 15 niñas, de origen caucásico, de los cuales el $80 \%$ estaba entre los 6 y 12 años de edad y cuyo cuociente intelectual promedio era de 95 , con un rango de 75 a 128 . Fueron excluidos niños con disfunciones neurológicas abiertas, como parálisis cerebral, epilepsia y enfermedades degenerativas del Sistema Nervioso Central. Fueron incluidos tres nin̄os con descargas epileptiformes en el electroencefalograma, pero sin episodios paroxísticos clínicos.

Solamente diez niños habían recibido drogas estimulantes, antes del estudio, administradas en dosis bajas y por periodos breves $(5$ recibieron anfetaminas y 5 metilfenidato). La edad ósea fue determinada evaluando radiografias de carpo, interpretadas dos veces, la segunda sin otro antecedente que la edad cronológica y sexo de los niños, comparándolas con las normas de maduración esquelética de Greulich y Pyle, consideradas perfectamente adecuadas para nuestro material. El peso y talla de los 60 niños fueron comparados con las normas de Stuart ${ }^{(13)}$ y los perímetros craneanos con las de Nellhaus. ${ }^{(14)}$ Las diferencias encontradas fueron sometidas a análisis estadístico, utilizando la prueba de $\mathrm{T}$ de Student.

\section{RESULTADOS}

De los 60 niños, seis $(10 \%)$ presentaron edades óseas significativamente retrasadas $\left(\begin{array}{lll}-2 & 1 / 2 & \text { a }\end{array}\right.$ -3 1/2 desviaciones standard) en relación a sus edades cronológicas. Un niño presentó edad ósea avanzada. Las manifestaciones de disfunción cerebral mínima que presentaban los seis eran las esperables para todo el grupo de acuerdo a nuestro criterio diagnóstico, y no mostraban otras anormalidades. Ninguno de ellos había sido tratado con anfetaminas o metilfenidato. De los seis, cinco tenían talla, peso y perímetro craneano dentro de límites normales.

La comparación entre las edades ósea y las edades cronológicas en los 60 niños mostró los siguientes hechos: 39 (65\%) tenían sus edades óseas bajo la media y sólo $11(18,3 \%)$ sobre ésta. La edad cronológica promedio de todo el grupo fue de 8 años 6 meses y la edad ósea promedio fue de 8 años. Esta diferencia es estadísticamente significativa (T:4.311; P:0.001). GRAFICO 1.

El peso, talla y perímetro craneanos del grupo estuvo dentro de límites normales. Seis niños presentaron estaturas superiores al percentil 97 y dos, inferiores al percentil 3. Cinco niños tenían pesos superiores al percentil 97 y sólo uno inferior al percentil 3. Cuatro tenían perímetros craneanos sobre el percentil 97 y dos bajo el percentil 3. Excepto pör la correlación radiologica de las circunferencias craneanas, las radiografías de cráneo fueron normales. El nivel de T 4 en los niños bajo el percentil 3 en peso, talla y perímetro craneano fue normal.

No se demostró que existieran algunos modelos o agrupaciones de síntomas y signos de disfunción cerebral mínima que pudieran correlacionarse con inmadurez esquelética o con déficit de crecimiento somático.

\section{DISCUSION}

La razón por la cual niños con seria disfunción o daño cerebral presentan frecuentemente retraso de su maduración esquelética es todavía poco clara. Se ha postulado ${ }^{(15)}$ que existiría una disfunción del eje hipotálamo-hipofisiario, pero esto no ha sido comprobado. Siendo de interés conocer si formas leves de disfunción del Sistema Nervioso Central se asocian también a retardo de la maduración ósea, hemos confirmado que los pacientes con disfunción cerebral mínima, como grupo, presentan un retardo significativo, mientras su peso, talla y perímetros craneanos tienen una distribución normal.

Si bien la precisión de las normas de Greulich y Pyle para niños norteamericanos de clase media y de origen anglosajón ha sido confirmada recientemente, ${ }^{(16,}{ }^{17)}$ idealmente deberían constituirse grupos de control, con niños pareados según edad, sexo y clase socioeconómica en la población local, para establecer comparaciones con el crecimiento pondoestatu- 
ral y maduración ósea de aquellos con disfunción cerebral.

\section{GRAFICO 1:}

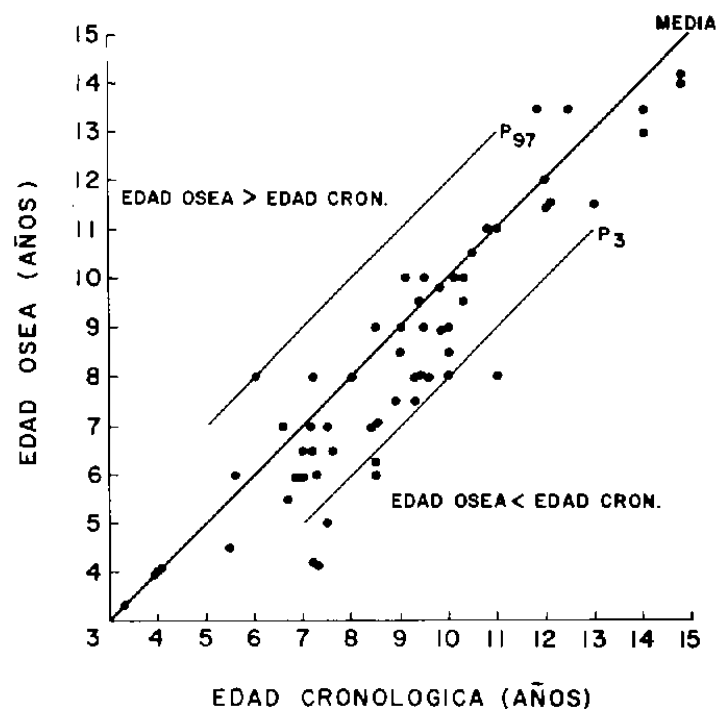

EDAD CRONOLOGICA MEDIA : 8.6 AÑOS

EDAD OSEA MEDIA: B AÑOS

$M_{\triangle}: 0,6$ AÑOS

Comparación entre las edades óseas y edades cronológicas en 60 niños con disfunción cerebral mínima.

\section{RESUMEN}

Se estudió el crecimiento pondoestatural y la maduración esquelética con radiografías de carpo en 60 nin̄os (45 niños, 15 niñas) que presentaban disfunción cerebral minima de acuerdo al criterio de Clements y Crichton. La mayoría tenía edades comprendidas entre 6 y 12 años. Todos tenían peso, talla y perímetro craneano esencialmente normales. Al interpretar las radiografías de acuerdo al atlas de Greulich y Pyle, se encontró que seis (10\%) tenían edad ósea significativamente bajo la media correspondiente a su edad cronológica, y sólo uno tenía edad ósea avanzada. La edad ósea media para el grupo fue de 8 años y por lo tanto significativamente por debajo de la edad cronológica media de 8 años 6 meses. Se discuten estos hallazgos en lo referente a disfunción cerebral y retardo de la maduración ósea y a la aplicabilidad local de las normas de Greulich y Pyle.

\section{SUMMARY}

Sixty Caucasian Western Canadian children ( 45 boys, 15 girls) with minimal brain dysfunction, mostly aged 6 to 12 years, had essentially normal measurements for height, weight and head circumference. The bone age was determined by X-Ray examination of hand and wrist, interpreted according to the Atlas by Greulich and Pyle (1959). Six (10\%) of the 60 children had a bone age significantly below the normal mean for their chronological age, and only one had a bone age wich was significantly advanced. The mean bone age for the whole group was 8.0 years and thus significantly low for the mean chronological age of 8.6 years. The findings are discussed with reference to brain dysfunction affecting skeletal maturation and the local applicability of Greulich-Pyle norms.

\section{REFEHENCIAS}

${ }^{1}$ Tobis, J.S. Saturen, P. Larios, G. y Posniak, A.O. Study of growth patterns in cerebral palsy. Arch. Phys. Med. Rehab. 42: 475,1961 .

${ }^{2}$ Ruby,D.O., Matheny, W.D. Comments on growth of cerebral palsied children. J. Amer. Diet. Ass. 40: 425, 1962.

${ }^{3}$ Kugel, R.B. y Mohr, J. Mental retardation and physical growth. Amer. J. Mental Def. 68: 41, 1963.

${ }^{4}$ Schmid, F. Das Handskelett bei früh infantilen Affektionen des Zen tralnervensystems. Fortsch. Geb. Roent. Nuklearmed. 86: 239, 1957.

${ }^{5}$ Geisler, E. y Bannes, M. L. Das Roentgenbild des Handskeletts als Hilfamittel zur Diagnostik zerebraler Schäden von Kindern. Münch. Med. W. 102: 1273, 1960.

${ }^{6}$ Lyttkens, G. y Persson, R. Kritische Beurteilung des Zusammenhanges zwischen Handskelett und Hirnschaden. Ann. Paediat. 206: 333, 1966 .

${ }^{7}$ Oettinger, L., Majouski, L. V. Limbeck, G.A. y Gauch, R. Bone age in children with minimal brain dysfunction. Percep. Motor Skills. 39: 1127, 1974.

${ }^{8}$ Clements, S.D. Minimal brain dysfunction in children. Monogr. 3. U.S. Dept. of Health, Ed. \& Welfare. Washington, D.C. 1966.

${ }^{9}$ Greulich, W. y Pyle, S.I. Radiographic Atlas of skeletal development of hand and wrist. Second Ed. Stanford Univ. Press, Stanford, Cal. 1950.

${ }^{10}$ Safer, D., Allen, R. y Barr, H. Depression of growth in hyperactive children on stimulant drugs. New Engl. J. Med. 287: $217,1972$.

${ }^{11}$ Safer, D. y Allen, R. Factors influencing the suppresant effects of two stimulant drugs on the growth of hyperactive children. Pediatrics 51: 660, 73

12 Crichton, J.U. Kendall, D.C. Catterson, J. y Dunn, H.G. Learning disabilities. Canadian Paediatric Soc. Sherbrooke, Quebec, 1972.

${ }^{13}$ Stuart, H.C. y Meredith, H. Normal growth and development. Am. J. Pub. Health 36: 1946.

${ }^{14}$ Nellhaus, G. Composite international and interracial graphs. Pediatrics 41: 106, 1968.

15 Mosier, H.D. Grossman, H.J. y Dingman, H.F. Secondary sex development in mentally deficient individuals. Child develop. 33: 273, 1962.

${ }^{16}$ Mellits, E.D. Dorst, J.P. y Cheek, D.B. Bone age, its contribution to the prediction of maturational or biological age. Am. J. Phys. Anthrop. 35: 381, 1971.

${ }^{17}$ Skeletal maturation of children aged six to eleven. National Health Survey, Series 11, nr. 149. In: Clin. Pediatrics 15: $569,1976$. 